\title{
Didactiques et/ou didactique. Poursuivre le travail de problématisation
}

Florence Ligozat, Maryline Coquidé, Corinne Marlot, Ingrid Verscheure et Gérard Sensevy

\section{(2) OpenEdition}

1 Journals

Édition électronique

URL : http://journals.openedition.org/educationdidactique/1907

DOI : 10.4000/educationdidactique.1907

ISSN : 2111-4838

Éditeur

Presses universitaires de Rennes

Édition imprimée

Date de publication : 30 juillet 2014

Pagination : 101-115

ISSN : 1956-3485

\section{Référence électronique}

Florence Ligozat, Maryline Coquidé, Corinne Marlot, Ingrid Verscheure et Gérard Sensevy,

" Didactiques et/ou didactique. Poursuivre le travail de problématisation », Éducation et didactique [En ligne], 8-1 | 2014, mis en ligne le 15 septembre 2016, consulté le 01 mai 2019. URL : http://

journals.openedition.org/educationdidactique/1907; DOI : 10.4000/educationdidactique.1907 


\section{DIDACTIQUES ET/OU DIDACTIQUE POURSUIVRE LE TRAVAIL DE PROBLÉMATISATION}

Florence Ligozat, GREDIC, FPSE, Université de Genève Maryline Coquidé, STEF, ENS Cachan E Institut Français d'Éducation ENS Lyon

Corinne Marlot, ACTé, ESPE d'Auvergne, Université Blaise Pascal

Ingrid Verscheure, UMR EFTS, Université de Toulouse le Mirail Gérard Sensevy, ESPE de Bretagne, Université de Bretagne Occidentale 
Florence Ligozat et al.

\section{Préambule}

Au cours de la préparation de ce dossier, le besoin s'est fait sentir de rassembler un certain nombre d'idées-forces et de questions vives qui parcourent, explicitement ou en filigrane, les différents points de vue et réactions, en distinguant des points de divergence et de convergence entre diverses contributions. Les lignes qui suivent tentent de montrer en quoi un espace de problématisation s'est ouvert, de notre point de vue. L'objectif principal de ce dernier développement du dossier est de soutenir la poursuite des débats, dans le cadre de la rubrique "Expression» de la revue Éducation \& Didactique, par exemple, et dans d'autres lieux.

\section{UNE ENTRÉE EN MATIÈRE : LE CAS DE LA CRISE EN PSYCHOLOGIE}

Dans leur présentation de l'édition française de l'ouvrage de L. S. Vygotski, « La signification historique de la crise en psychologie », paru en 1999, Jean-Paul Bronckart et Janette Friedrich retracent le contexte historique et les fondements philosophiques des « écoles de psychologies » de la première moitié du XX ${ }^{e}$ siècle en Europe. Ces écoles, disent-ils, ont coïncidé avec l'avènement de différents projets d'unification du champ, sous l'angle d'une psychologie générale, dont celui de L. S. Vygotski proposant une psychologie unifiée de l'activité comportementale et mentale humaine. En guise de vignette introductive à la problématique du rapport entre les "didactiques » et une possible "didactique ", nous proposons ci-dessous un bref résumé du point de vue de ces auteurs.

À l'issue d'un inventaire des différents courants de travaux en psychologie, révélant une partition entre les tenants d'une «science de la nature » et les tenants d'une « science de l'esprit » dans les années 20, JeanPaul Bronckart et Janette Friedrich commentent : «à l'évidence, l'éclatement de la discipline $e^{1}$ est la conséquence de divergences d'options épistémologiques ayant trait au statut des phénomènes psychiques ainsi qu'à celui des ceuvres humaines sensées (langage, structures sociales, cultures, etc.) ». Ils ajoutent qu' « en dépit des débats et interrogations, la majorité des psychologues semblent - déjà - adopter une attitude de méfiance attentiste à l'égard des questions épistémologiques ; à leurs yeux, il est inutile de continuer à se poser ce type de question au plan conceptuel ou proprement théorique; les données des recherches scientifiques suffiront un jour à les résoudre » (ibid. p. 23-24). Dans le cas « des psychologies», c'est la discordance entre les approches théoriques, développées au sein de l'académie d'une part et les approches praticiennes d'intervention d'autre part, qui va révéler une « crise » et interroger ce que pourrait ou devrait être le champ de la psychologie. Pour autant, les auteurs remarquent que la brièveté du débat qui s'est alors ouvert ne permettra pas de résoudre la crise en profondeur, autrement que par des cohabitations entre démarches «naturaliste » et « humaniste», par des clarifications entre intervention pratique (psychologie clinique) et investigation scientifique (psychologie expérimentale), visant à conserver différentes approches au sein de chaires dites de "psychologie générale». Les perspectives de solutions intégratives, telles celles proposées par Karl Bühler, Georges Politzer ou Lev S. Vygotski, sont passées quasiment inaperçues, pour des raisons diverses. Les auteurs notent qu'en conséquence, le fractionnement s'est poursuivi et s'est même "solidifié » dans la période d'aprèsguerre, avec une psychologie académique caractérisée par la coexistence de sous-disciplines disjointes, centrées sur des objets spécifiques et revendiquant des méthodologies propres : psychologie expérimentale (de l'adulte), psychologie du développement (de l'enfant), psychologie sociale, clinique, scolaire, des émotions, du langage, etc. Jean-Paul Bronckart et Janette Friedrich poursuivent : "il n'est guère étonnant dès lors que les certitudes behavioristes se soient effondrées comme châteaux de cartes sous l'effet de la révolution cognitiviste, et que cette dernière se soit déployée dans une confusion épistémologique sans égal, en se situant objectivement dans le prolongement du béhaviorisme médiationiste (dans un projet de "naturalisation de l'esprit " très proche de celui de Hebb), tout en postulant une inversion des rapports entre dimensions comportementales et mentales (dans l'affirmation du modularisme selon laquelle "la structure mentale cause la structure des comportements »-Fodor, 1986, p. 13)» (ibid. p. 34).

Malgré un intérêt certain, il ne saurait être question ici, de développer une comparaison de la situation « des psychologies» (de « la »psychologie) dans les années 20 avec celle « des didactiques» (de « la» didactique) aujourd'hui. Ces champs scientifiques, au delà du fait qu'ils relèvent tous deux des sciences humaines et sociales, ont des connexités 
Florence Ligozat et al.

indéniables. Peut-on étudier la nature et l'évolution des « contenus », des « savoirs » ou, plus largement, des œuvres culturelles faisant l'objet d'un enseignement/apprentissage, sans penser la manière dont les acteurs de l'enseignement et de l'apprentissage les font vivre dans les structures sociales ad hoc ? Et de quelle manière ces acteurs se construisent en tant que personnes, dans les différents rapports qu'ils sont amenés à établir aux éléments de la culture sous couvert d'un curriculum? Nous aurons l'occasion de revenir sur ces questions un peu plus loin.

Nous pensons que certains éléments de l'évolution historique et épistémologique des psychologies versus de la psychologie qui viennent d'être décrits peuvent avoir une valeur heuristique dans la manière d'envisager les rapports entre " didactiques » et « didactique ». En somme, comment la communauté des didacticiens pourrait-elle tirer partie des aléas de l'histoire de la solidification « des psychologies» pour (mieux) penser le développement de son propre champ de recherches ? Cette question concerne notamment la quête des moyens de dépassement du cloisonnement des didactiques, que l'ensemble des contributeurs de ce dossier pointe, voire déplore, comme une source de fragilité épistémologique et/ ou institutionnelle du champ.

En substance, du cas de la « crise en psychologie », nous retenons trois éléments susceptibles de nous faire réfléchir sur des conditions de dépassement du cloisonnement :

- il est des « crises » ou des signes, dans le rapport des théories et des modèles produits aux problèmes du monde réel, qui doivent rendre les chercheurs attentifs à la nécessité de l'évolution du ou des domaines de recherche concernés ;

- les solutions " oecuméniques », visant à ménager et rassembler toutes les tendances en un pseudo-champ unifié, sans questionner les différences, les spécificités, les objets communs, les rapports de complémentarité ou de contradiction entre les différentes approches revendiquées, contribuent à un morcellement du champ, par une institutionnalisation des cloisonnements ;

- faire l'économie de l'identification des courants épistémologiques qui structurent le champ ne permet pas d'identifier ses points de fragilité potentielle et fait prendre un risque d'émergence de nouveaux courants dominateurs, marginalisant ou pervertissant certains principes fondamentaux du champ.
Ces trois éléments peuvent (1) apporter un éclairage, nouveau ou différent, sur certaines orientations qui émergent, en plein ou en creux, dans le dossier ; (2) permettre de dégager quelques conséquences possibles pour la dynamique des rapports entre « didactiques » et " didactique », dans le cadre de la problématisation recherchée.

\section{DIDACTIQUES ET/OU DIDACTIQUE : LES CONTOURS D'UN CHAMP DE RECHERCHE}

\section{Une nouvelle strate de réflexion}

Le moment de l'émergence du comparatisme en didactique, à la fin des années 90, s'est manifesté par des tentatives de construire des rapports entre:

- les disciplines scolaires, par exemple à travers la maîtrise d'outils sémiotiques et langagiers dans différentes disciplines (Colomb \& Martinand, Éd., 2000) ;

- les concepts et les didactiques (des disciplines $)^{2}$, par exemple le contrat didactique, la transposition didactique, le rôle de la médiation enseignante dans les différentes didactiques (Raisky \& Caillot, Éd., 1996);

- le spécifique et le générique dans les pratiques d'enseignement lapprentissage, en recensant et en éprouvant des catégories candidates ayant émergé dans telle ou telle approches, au sein des didactiques (Venturini, Amade-Escot \& Terrisse, Éd., 2002 ; Mercier, Schubauer-Leoni \& Sensevy, Éd., 2002).

Ces travaux, engageant des mises à l'épreuve empiriques pour l'essentiel, contrastent avec les points de vue développés aujourd'hui dans ce dossier. De tentatives visant à combler des lacunes d'approches didactiques dites " cloisonnées », parce que historiquement développées dans le prolongement de disciplines (ou matières) scolaires, on constate qu'il est désormais possible de débattre de ce que peuvent ou doivent être les composantes fondamentales d'un champ de recherches scientifiques constitué par « les didactiques (des disciplines)», "des approches didactiques (de pratiques d'enseignement/apprentissage, du curriculum, de la formation professionnelle, etc.) », ou encore l'étude du didactique « tout court».

Cela ne veut pas dire que les travaux empiriques ont disparu, tout au contraire, comme en témoignent l'actualité des publications telles que "Les didactiques en question(s) : état des lieux et perspectives 
Florence Ligozat et al.

pour la recherche et la formation » (Elalouf, Robert, Belhadjin, Bishop, Éd., 2012), « Les contenus disciplinaires. Approches comparatistes » (Daunay, Reuter \& Thépaut, Éd., 2013) ou encore "Didactique en construction, constructions des didactiques " (Dorier, Leutenegger \& Schneuwly, Éd., 2013). Mais parallèlement, un débat s'est ouvert pour identifier certains marqueurs irréductibles du champ des recherches " à caractère didactique », avec l'idée d'arriver à définir ce qui relève du champ et ce qui renvoie à ses limites. Quel que soit la dénomination retenue pour ce champ, le débat dont rend compte ce dossier a la propriété fondamentale de se situer au-delà des particularismes de telle ou telle discipline ou ensemble de pratiques, ce qui est une avancée en soi. Ceci n'a, du reste, pas échappé à nos discutants, Patrick Rayou et Christian Orange. Lorsque le premier précise que " cette riche journée d'étude a montré des volontés de dialogue, sinon d'unification, entre des approches diverses et parfois contradictoires de la didactique » (Rayou, dans ce numéro), le second - se positionnant en didacticien de la biologie, intitule sa contribution : «Unité et diversité de la didactique » (Orange, dans ce numéro).

Trois modes d'entrée dans l'espace des recherches en didactique, non exclusives, sont repérables dans les différentes interventions.

1. Une entrée par rapport aux contenus, qu'ils soient identifiables en termes de pratiques, de dispositions, de valeurs, de compétences ou de savoirs ; qu'ils s'organisent en curricula, en matières ou en disciplines scolaires ou qu'ils relèvent d'objets frontières ou composites issus de différentes sphères d'activités sociales. Ces approches questionnent l'évolution historique, l'épistémologie et les références des contenus dans les curricula et les pratiques. Cette entrée est présente dans les points de vue de Yves Reuter, Jean-Louis Martinand, Bernard Schneuwly, Helmut Johannes Vollmer et dans la discussion de Christian Orange.

2. Une entrée par rapport aux visées des recherches en didactique(s), qui peuvent se traduire par différentes postures des chercheurs vis-à-vis des pratiques d'enseignement et apprentissage et leurs contenus. C'est la tension entre les dimensions descriptive / critique d'une part et technologique / normative d'autre part des recherches en didactique(s) qui est plus particulièrement invoquée par Jean-Louis Martinand et aussi par Bernard Schneuwly, à travers les rapports complexes entre champ scientifique et champ professionnel.

3. Une entrée par rapport aux pratiques sociales d'enseignement et apprentissage (le didactique) dont les finalités sont la diffusion / transmission de savoirs (en tant que pratiques sociales « transposées »), s'exerçant dans des structures institutionnelles programmatiques ou, plus largement, dans l'ensemble des relations sociales où se jouent des mouvements vecteurs de culture. Cette entrée, de nature anthropologique et historico-sociale, est présente dans les points de vue de Yves Chevallard, Bernard Schneuwly et dans la discussion de Patrick Rayou.

\section{La sémantique du terme « didactique(s)»: un} enjeu conceptuel

Dans la délimitation des contours du champ des recherches didactiques, la sémantique des termes utilisés dans chaque point de vue est important : didactique en tant qu'adjectif d'une part et la didactique, les didactiques, le didactique, en tant que noms communs, d'autre part. Le statut et la vocation de la (des) didactique(s) peuvent être en cause, un même terme peut cependant référer à des réalités différentes selon les points de vue développés.

Les didactiques: Jean-Louis Martinand et Yves Reuter envisagent des didactiques. Dans la mesure où les enseignants enseignent des disciplines du second degré ou des matières éducatives au primaire, les didactiques « de discipline » (Martinand, dans ce numéro) comme domaine de recherche constituent, de fait, un champ d'investissement pour une partie des formateurs. Yves Reuter considère d'ailleurs le terme de " didactique disciplinaire " comme une tautologie, tandis que pour Jean-Louis Martinand, les significations du terme « didactiques » sont plus hétérogènes, suivant que des didacticiens s'intéressent à l'école primaire ou à la formation professionnelle, par exemple. Pour Yves Reuter la préoccupation centrale de recherche d'une didactique est de "travailler à éclairer les problèmes et les situations rencontrés par les élèves et de penser des pistes pour des solutions possibles à partir des contenus et des disciplines scolaires " (Reuter, dans ce numéro). Jean-Louis Martinand, de son côté, met en avant une forme de polyvalence des didactiques par rapport à leurs propres champs d'intervention : didactique en tant qu'activités de 
Florence Ligozat et al.

recherche, didactique pour qualifier des activités de formation, didactique en tant qu'approches normatives pratiquées par les cadres de l'éducation, didactique aussi bien pour l'enseignement scolaire, voire de formation ou d'éducation hors écoles.

Par ailleurs, Jean-Louis Martinand développe une conception élargie du champ des didactiques à l'ensemble des activités curriculaires. Il met en avant une nécessité de penser un programme didactique en termes de curricula et non uniquement de disciplines scolaires, surtout dans la forme actuelle de celles-ci.

Une comparaison des points de vue de Bernard Schneuwly et Yves Chevallard amène à la production de deux termes et notions liés, la didactique, et le didactique. La didactique: Bernard Schneuwly et Yves Chevallard parlent tous deux d'une « didactique " tout court, mais chacun ne lui attribue pas le même domaine de réalité, à juger de la restriction posée par le premier sur les conditions d'existence d'une relation didactique - "dans des institutions spécialisées », c'est-à-dire des espaces sociaux spécifiques de la transmission des savoirs, pour Bernard Schneuwly (Schneuwly, dans ce numéro)- en regard de l'ouverture envisagée par le second : " dès lors qu'une instance $U$ (et par instance on entend ici une personne ou une institution) fait quelque chose [...] pour qu'une instance $V$ intègre à son équipement de savoir et de savoir-faire [...] certaines connaissances [...] » pour Yves Chevallard (Chevallard, dans ce numéro).

Le didactique: ce terme est proposé de longue date par Yves Chevallard (Chevallard, 1985/1991) pour désigner l'ensemble des faits sociaux liés aux pratiques d'enseignement et d'apprentissage. Il est également utilisé par Bernard Schneuwly, et on le retrouve aussi dans le courant des recherches de didactique comparée, comme en témoigne la discussion animée par Francia Leutenegger : «l'existence de chaires de didactique comparée est intimement liée également à des projets collectifs où différents spécialistes de disciplines ouvrent ensemble, au sein d'une même équipe, avec la particularité de se doter de cadres conceptuels plus unifiés pour étudier LE didactique » (Leutenegger, dans ce numéro).

\section{Un besoin de cohésion face à de nouvelles dynamiques institutionnelles}

Les nombreux changements, qui se sont produits ces dernières années sur le terrain d'étude des didac- ticiens, ne sont sans doute pas anodins quant à la possibilité de construire aujourd'hui les contours d'un champ de recherches scientifiques en didactique(s). La question de l'amélioration des conditions d'accès aux savoirs, - contemporaine de la démocratisation des études secondaires, et moteur historique du développement des didactiques (des disciplines) dans les années 70-90 - est aujourd'hui concurrencée par de nouveaux de enjeux sociétaux qui modifient les pratiques scolaires.

Au niveau du curriculum, de nouveaux objets ou contenus sont apparus tels que "les éducations à " (l'environnement, le développement durable, la santé, la sécurité, la communication, etc.). De nouvelles exigences sont posées dans les connaissances que les élèves doivent manifester (par exemple, l'articulation savoirs - compétences), en même temps que de nouveaux dispositifs, pluri- ou codisciplinaires ont été mis en ouvre (Travaux personnalisés encadrés, Parcours croisés, Itinéraires de découvertes, dans le cas de la France), et des mouvements de compartimentation / décompartimentation ou de reconfiguration des domaines disciplinaires sont constatés ${ }^{3}$. Ces changements concernent aussi des pratiques institutionnelles, avec des injonctions telles que la mise en œuvre de démarches d'investigation scientifiques (inquiry-based science teaching/learning) promue par des standards internationaux pour enseigner les disciplines scientifiques (Rocard et al., 2007).

Ces nouveaux objets, ces déplacements ou ces nouvelles organisations, entre disciplines, entre dispositifs, et entre les responsabilités des acteurs, posent des questions inédites aux didacticiens. Ceux-ci sont conduits à coopérer pour construire de nouvelles problématiques, chercher ensemble des réponses, sur la base de leurs compétences respectives. À quels savoirs ou pratiques de référence sont adossés ces nouveaux objets? À quelles conditions, ces exigences du curriculum peuvent-elles être satisfaites dans les pratiques de classe? Comment identifier ce qui est effectivement enseigné et appris dans ces nouveaux dispositifs de travail ? Comment interpréter les difficultés rencontrées dans les mises en œuvre? Autant de questions qui semblent difficiles à traiter depuis les seuls cadres épistémologiques de quelques disciplines bien délimitées. Ce sont des faisceaux d'approches qu'il semble alors nécessaire de combiner pour nourrir une dialectique entre des sciences didactique(s) qui traitent de l'épistémologie des contenus scolaires et une "science didactique " 
Florence Ligozat et al.

qui contribue à étudier l'évolution du projet d'éducation / instruction d'une société.

La question de l'efficience de l'école est aussi plus que jamais débattue dans une société qui tente de rationnaliser ses investissements en même temps qu'elle se doit de réduire l'échec scolaire et améliorer la formation de tous. Ainsi, la mise en place de dispositifs d'accompagnement des élèves en difficulté, tout comme les réformes de la formation des enseignants, sont des dynamiques institutionnelles dont les didacticiens, en collaboration avec d'autres approches des sciences humaines et sociales (sociologie, ergonomie, sciences de la communication, etc.) ont tout intérêt à se saisir ; tout d'abord parce qu'elles ont des effets importants sur les savoirs enseignés et appris dans les classes mais aussi parce qu'elles donnent à voir - comme le ferait un miroir grossissant - les préoccupations, les tensions et les dilemmes qui traversent la profession enseignante aujourd'hui. Ces derniers sont d'autant plus importants à identifier qu'ils constituent l'arrière-plan des formes d'action didactique. La complexité de ces dynamiques exige des approches globales qui ne peuvent se réduire à l'analyse des conditions spécifiques de l'enseignement /apprentissage de tel ou tel contenu. Ces approches reposent, entre autres, sur une possibilité de distinguer et d'articuler les dimensions spécifiques versus génériques des pratiques d'enseignement par rapport aux exigences épistémologiques des savoirs enseignés.

Dans cette perspective, la recherche d'une plus grande cohésion entre différents courants ou domaines de recherche en didactique(s) peut être lue comme une tentative de réponse à des limites théorico-méthodologiques révélées par les dynamiques des réalités étudiées, et donc comme une recherche de moyens de dépassement d'une « crise » face aux mutations du terrain.

\section{DIDACTIQUE ET/OU DIDACTIQUES : QUELQUES POINTS AVEUGLES}

\section{Quid des logiques d'acteurs?}

Dans les lignes qui suivent, nous avons sélectionné des éléments de définition de la /les didactique(s), produits par les différents auteurs qui se sont risqués à cet exercice. À l'exception de la définition de Helmut Johannes Vollmer, qui cible les « manières d'enseigner » un contenu et la
« formation des êtres humains » à travers la notion de «Bildung », les définitions apportées tendent à désigner une science de la culture qui se déploie dans l'école (et au-delà selon Yves Chevallard), faisant appel à des notions clés telles que des " contenus », des " objets enseignables », des "savoirs», des " équipements praxéologiques », des " matières", des « disciplines », etc.

- Bernard Schneuwly : " Les didactiques constituent un champ de recherche scientifique ayant pour objet la transmission - vermittlung (médiation)- de savoirs dans des institutions spécialisées à cette fin » (Schneuwly, dans ce numéro);

- Helmut Johannes Vollmer: " the German term Didaktik, independent of its historical roots and shades of meaning, traditionally denominates the science of teaching and learning, relating to the formation of human beings and thus to any content or content-based skills inside and outside school. [...] All this issues are dealt with by specific domains called Fachdidaktiken (in the plural): the term thus relates to the science and art of teaching and learning subject-specific content, skills and procedures inside and outside school. » (Vollmer, dans ce numéro);

- Yves Chevallard : " La didactique est la science dont l'objet premier est le didactique, c'est-à-dire l'ensemble des faits didactiques. Il y a fait didactique (ou geste didactique) dès lors qu'une instance $\mathrm{V}$ (et par instance on entend ici une personne ou une institution) fait quelque chose ou même envisage de faire quelque chose pour aider à faire qu'une instance $U$ intègre à son équipement de savoir et de savoir-faire - à son équipement praxéologique- certaines connaissances relatives à l'enjeu du geste didactique » (Chevallard, dans ce numéro);

- Yves Reuter : « je préfère - nous préférons au sein de Théodile- employer le terme « didactiques " au pluriel, en relation avec les disciplines scolaires et la manière dont nous interrogeons la notion de disciplines » (Reuter, dans ce numéro) ;

- Jean-Louis Martinand : « les didactiques sont avant tout des disciplines technologiques, des sciences de l'ingénierie culturelle [...] leur spécificité est l'exercice d'une responsabilité sur les contenus » (Martinand, dans ce numéro);

- Christian Orange : " Les didactiques étudient les systèmes (les disciplines) - systèmes 
Florence Ligozat et al.

dynamiques, avec histoire et mémoire - produisant de l'enseignable dans des institutions dédiées » (Orange, dans ce numéro).

Aux extrêmes, figurent le point de vue de Yves Reuter d'une part, et celui d'Yves Chevallard d'autre part. Le premier défend l'existence « des didactiques » par rapport à la notion de discipline scolaire et leur pluralité. Mais la notion de « didactique » peut-elle se réduire à celle de «discipline » ? Le deuxième défend l'existence " du didactique » comme fait social partout dense dans les interactions humaines, non nécessairement régies par des organisations disciplinaires, ni mêmes scolaires. Mais quelles sont alors les limites du champ de « la didactique $»$ ?

Dans les diverses contributions, l'éclairage apporté par Patrick Rayou depuis la sociologie de l'éducation fait longuement référence aux situations d'apprentissage, et la manière dont les savoirs prennent sens pour les acteurs des systèmes scolaires (les élèves principalement), comme un objet d'étude relevant à la fois de la sociologie et de la didactique. Cela rejoint une préoccupation d'Yves Reuter, développée sous l'angle de la « conscience disciplinaire » des élèves, en tant que construction d'un rapport au savoir disciplinaire dans les manières de penser, de parler et d'agir propres à la discipline en question. Cela rejoint aussi l'idée de " discipliner » les élèves (au sens d'une enculturation dans un domaine spécifique) et renvoie au rapport de l'enseignant à la discipline (ou matière) qu'il enseigne. Dans le point de vue d'Yves Chevallard, la place des acteurs dans la diffusion des savoirs existe dans la saisie « du didactique " comme l'intervention d'une instance (institution ou personne) sur une autre instance à propos d'un enjeu, mais les modalités de cette intervention ne sont pas précisées. Dans les points de vue de JeanLouis Martinand, Bernard Schneuwly et Christian Orange tels que développés dans ce dossier, les logiques d'acteurs semblent absentes de l'espace de problématisation en construction.

Ce constat nous ramène à l'une des premières questions que nous avons soulevée à propos des connexités entre le champ des psychologies (de la psychologie) et celui des didactiques (de la didactique). Le champ des recherches en didactique(s) pourrait-il se passer d'être aussi, une science des êtres humains qui portent, transmettent, acquièrent, transforment la culture ? Et, par ricochet, les communautés de didacticiens peuvent-elles se passer d'intégrer ou d'interagir avec des chercheurs non directement spécialistes des disciplines scolaires, mais inscrits plus largement dans les sciences humaines et sociales connexes?

Sans mettre en doute le bien-fondé des points de vue des auteurs cités, il nous semble important de souligner qu'une catégorisation aussi marquée des didactiques (la didactique) comme science(s) des objets de la culture, prend le risque de faire perdre de vue que l'enseignement et l'apprentissage relève aussi des sciences de ceux qui agissent au sein des systèmes de transmission de cette culture. Parce qu'il ne saurait y avoir des objets construits et transmis en dehors de toute activité humaine, et que toute volonté d'intervention sur les contenus ou savoirs enseignés passe par une intervention sur les pratiques, et donc sur des formes d'action humaines. Il existe certes des chercheurs spécialistes de l'analyse de l'activité humaine dans les sciences dites « de l'Homme et de la société » qui s'intéressent aux logiques des acteurs dans les systèmes d'éducation et de formation, mais le champ des recherches en didactique(s) peut-il se satisfaire d'une juxtaposition épistémologiquement faible entre l'étude des objets de la culture d'une part et celle du fonctionnement des acteurs de la relation didactique d'autre part?

De notre point de vue, le champ des recherches en didactique(s) a tout intérêt à prendre à bras-le-corps une certaine dichotomie implicite entre « sciences des objets » et «sciences des sujets ", afin de construire un point de vue original et spécifique sur l'activité de transmission / acquisition de la culture, au sein des sciences de l'Homme et de la société. Ne pas entrer en matière sur cette construction, c'est laisser la voie libre à des approches de l'enseignement, de l'apprentissage et des pratiques scolaires plus généralement, dans lesquelles la spécificité des contenus ou savoirs n'est pas prise en compte, ou dans lesquelles l'activité par laquelle les savoirs prennent leur forme est minorée, voire ignorée.

\section{Quid des rapports aux sciences de l'Homme et de la société?}

Cela nous amène à questionner le rôle des disciplines connexes au champ des recherches en didactique(s) et certains présupposés épistémologiques apparaissant dans les points de vue de ce dossier. 
Florence Ligozat et al.

Globalement, tous les points de vue se font forts de discuter les rapports complexes entre didactique(s) et disciplines scolaires, mais aussi avec les disciplines apparentées aux objets scolaires. Pour Yves Reuter, ces disciplines ou domaines de pratiques apparentés constituent un espace de travail complémentaire du didacticien, qui se doit de maîtriser la discipline dont il entend construire la didactique. Ce point de vue semble également partagé par nos collègues allemands, chez qui la possibilité d'obtenir un poste académique lié à une Fachdidaktik repose sur l'exigence d'avoir enseigné la discipline scolaire et donc d'avoir une certaine spécialisation dans cette discipline (Vollmer, dans ce numéro). Un tel point de vue pose naturellement question vis-à-vis des nouveaux objets curriculaires et nouvelles pratiques scolaires que nous mentionnions précédemment. Quelle est alors la «discipline " qui doit être maîtrisée par le didacticien dans ces cas-là ? Une agrégation de biologie est-elle une formation plus pertinente qu'une agrégation d'EPS, pour problématiser les savoirs à enseigner dans le cadre de l'éducation à la santé ? Un diplôme d'infirmier pourrait-il être pertinent ? Faudrait-il construire une didactique spécifique de l'éducation à la santé, au risque de découper davantage le champ des recherches en didactique(s) au gré des dynamiques du terrain scolaire?

À l'opposé, pour Yves Chevallard, le rapport entre didactique(s) et disciplines apparentées aux savoirs scolaires ne va pas de soi, ouvrant la possibilité qu'un didacticien construise des objets d'étude en didactique à propos de savoirs, pour lesquels il n'a pas une formation disciplinaire "poussée ", c'est-à-dire dont il n'est pas personnellement spécialiste. Cela ne signifie pas qu'il n'y ait rien à savoir des disciplines pour mener une recherche didactique, mais que la spécialisation nécessaire se construit, en même temps que l'étude didactique elle-même, au sein du collectif qui la mène. Ce point de vue simplifie considérablement la prise en charge des dynamiques curriculaires dont nous parlions, mais sur le plan pratique, il comporte une exigence de taille : se faire "spécialiste » des éléments nécessaires à l'instruction d'une question nécessite d'explorer, entre autres, leurs configurations épistémologiques dans diverses strates disciplinaires (scolaire, universitaire, scientifique). Et il n'est pas certain que les conditions institutionnelles actuelles du développement des recherches en didactique(s) le permettent vraiment (Biagioli, dans ce numéro). Nous y revenons plus loin.
Le rôle des disciplines des sciences de l'Homme et de la société, auxquelles les didacticiens font des emprunts théoriques et méthodologiques, est en revanche beaucoup moins évoqué. Pour Yves Chevallard, les apports de ces disciplines sont nécessaires à l'enquête sur les niveaux de détermination des systèmes didactiques. Yves Reuter revient sur la nécessité de la conversion ${ }^{4}$ des focales habituellement investies par les « sciences des sujets » dans la problématique didactique (les affects, la communication, les relations sociales, l'expérience, etc.), sous l'angle de la conscience disciplinaire. JeanLouis Martinand insiste sur la nécessité d'équipes de recherche polyvalentes avec une variété de profils (psychologue, sociologue, linguiste, historien, etc.) pour éviter des dérives « auto-instituantes » de la part de didacticiens, dans ces domaines.

En quoi la collaboration avec les disciplines des sciences humaines et sociales, voire la conversion de certains concepts, permettent-elles de mieux comprendre des effets des choix éducatifs et scolaires de nos sociétés? À quelles conditions ce rapprochement est-il fécond?

Nous relayons ici le point de vue de Patrick Rayou, lorsqu'il dit que " la construction d'une socio-didactique, comme discipline nouvelle, ni sociologique ni didactique, morcellerait vraisemblablement à nouveau un paysage qu'elle ambitionne d'unifier » (Rayou, dans ce numéro). Il semble plus intéressant de construire une problématisation commune à partir de questions clairement identifiées dans les deux disciplines. Cette construction commune s'appuie sur la migration - d'un champ à l'autre- de certains concepts candidats. Il s'agit de produire des lectures renouvelées aussi bien des questions de recherche, que des résultats qui semblent "évidents » depuis une approche donnée. C'est ce qui se fait, par exemple, dans le cadre du réseau RESEIDA qui réunit des sociologues et des didacticiens sur la question de la construction et du renforcement des inégalités scolaires du point de vue des pratiques enseignantes. Afin de s'emparer de cette question, un ensemble de concepts, issus de la didactique comme de la sociologie, ont été éprouvés et ont permis de renouveler le questionnement aussi bien didactique que sociologique. Ainsi, comme le précise Patrick Rayou dans son texte, la rencontre, lors de l'analyse de corpus communs, de la notion de « contrat didactique différentiel » (Schubauer-Leoni, 1988) avec celles de «malentendu socio-cognitif » et 
Florence Ligozat et al.

« registre de travail des élèves » (Bautier et Rochex, 2007 ; Bautier \& Rayou, 2013) « appelle des re-et co-reproblématisations, dans lesquelles la didactique n'est pas seulement convoquée pour ses apports en matière de transposition du cognitif en scolaire, mais se trouve requise comme indispensable pour comprendre en profondeur ce qui se joue aussi dans l'élaboration des valeurs culturelles et des identités symboliques à l'école » (Rayou, dans ce numéro).

Le problème de la place des sujets/acteurs/agents de la relation didactique, tout comme celui des disciplines contributives au champ des recherches en didactique(s) nous paraissent devoir être pris au sérieux dans la construction de l'espace de problématisation qui nous occupe. Un manque de théorisation des logiques d'acteurs fait prendre le risque de développer des modèles de fonctionnement des savoirs dont les praticiens peinent à se saisir ${ }^{5}$, et peut accroitre un risque de coupure entre une science « académisée ${ }^{6}$ et son domaine de réalité. Qui plus est, un déficit d'échanges avec les communautés de chercheurs des sciences humaines et sociales n'incite pas à une réflexion élargie sur les fondamentaux du champ qui s'inscrivent nécessairement dans des logiques sociale, communicative, anthropologique, psychologique, etc. Paradoxalement, ce manque de perméabilité augmente un risque d'une domination épistémologique du champ des didactiques / de la didactique par ces mêmes disciplines, car ces dernières bénéficient d'une solide assise institutionnelle dans le monde académique, favorisant la confiance des organismes de financement nationaux et internationaux de la recherche.

\section{DIDACTIQUES ET/OU DIDACTIQUE : QUELS NOUVEAUX ENJEUX SE PROFILENT?}

La genèse historique du profil de « didacticien » et son évolution

En évoquant la question de la place des rapports de la didactique (des didactiques) aux sciences de l'Homme et de la société, la réflexion se porte aussi sur la genèse du (des) profils de didacticiens. Comment devient-on didacticien? Nous ne posons pas ici directement la question de la formation, mais celle de la différence des parcours, des Bildung spécifiques des chercheurs, en tant qu'acteurs dans ce domaine. Cet aspect est soulevé par Jean-Louis Martinand, qui revient sur son propre parcours et, d'une manière très différente, par Yves Chevallard qui souligne un inachèvement de la rupture épistémologique entre enseignants et didacticiens, qui se vivent ou sont perçus comme les « grands frères » des enseignants.

Tel chercheur a été formateur à l'école normale dès le début de sa carrière, dans une discipline spécifique, puis enseignant-chercheur en sciences de l'éducation (à l'intérieur de celle-ci, spécialiste de la didactique de cette discipline). Tel autre a été professeur dans l'enseignement secondaire quelques années, agrégé dans une discipline, puis didacticien de cette discipline, à l'intérieur de celle-ci. Tel autre encore, a été instituteur de longues années, puis est devenu didacticien au sein des sciences de l'éducation, en ayant développé des travaux à propos d'une « matière » enseignée à l'école primaire. Un quatrième a exercé en tant que « chercheur académique » avant de s'intéresser aux recherches en éducation. D'autres encore ont été formés dans certains domaines de la psychologie ou encore dans les sciences du langage et se sont impliqués dans l'avènement des recherches en didactique(s) dans les années 80 , aux côtés des précédents, en devenant, de fait, des didacticiens ${ }^{7}$.

Cependant, il faut admettre que la diversité des profils semble se restreindre, en même temps que se pose un problème de relève ${ }^{8}$. Désormais, un contingent non négligeable de didacticiens est formé à certaines spécialités didactiques (les mathématiques et les sciences en particulier) au sortir d'une formation dans une discipline académique, selon un parcours master et doctorat, sans passer (ou très peu) par une phase d'enseignement. Dans le même temps, les migrations ou conversions de chercheurs, formés dans les disciplines connexes des sciences de l'Homme et de la société, se tarissent. Ce double phénomène interroge l'affirmation de Jean-Louis Martinand, pour qui « les didactiques ne sont pas des disciplines autoreproductrices » (Martinand, dans ce numéro).

Ces parcours différents ne sont-ils pour rien dans les positions épistémologiques des chercheurs? Quelles sont les conséquences d'une tendance à l'uniformisation des profils de didacticiens? Il nous semble que différentes filiations de chercheurs en didactique(s) sont à encourager pour organiser la relève dans la communauté. Un premier principe pourrait être celui de la pratique effective et durable d'une fonction d'enseignement ; un second pourrait tenir à une orientation de niveau ou de filière : « école primaire ", « école secondaire », filière de formation 
Florence Ligozat et al.

générale, technologique ou professionnelle, etc. Un troisième principe pourrait être une responsabilité de formation d'enseignants. Un croisement de ces principes (sans doute avec d'autres) pourrait éclairer des originalités dans la genèse et les positionnements épistémologiques et théoriques des uns et des autres.

\section{DES RAPPORTS AMBIVALENTS AVEC LES INSTITUTIONS DE FORMATION}

En relation directe avec les points précédents, le rôle des structures de formation des enseignants qui orientent fortement la définition des profils de didacticiens est peu évoqué dans ce dossier, malgré une question explicite dans le texte d'invitation à cette journée (Cf.- la présentation du dossier par Florence Ligozat, Maryline Coquidé \& Gérard Sensevy dans ce numéro). Les structures de formation des enseignants (telles les Écoles Supérieure du Professorat et de l'Éducation en France, l'Institut Universitaire de Formation des Enseignants à Genève ou les Hautes Écoles Pédagogiques dans d'autres cantons suisses) alimentent une bonne part des heures d'enseignement ou de formation dans le service des didacticiens. À ce titre, elles jouent un rôle important dans le développement des recherches didactiques, par une voie qui semble naturelle et par conséquent peu questionnée : les exigences de spécialisation disciplinaires des intervenants dans la formation didactique des enseignants. Seul Yves Chevallard a engagé une réflexion sur ce point, à la suite d'un échange que nous avons eu avec lui, concernant les conditions de possibilités du didacticien « gyrovague » qu'il envisage.

Il est à noter que cette question a été abordée ailleurs par Chantal Amade-Escot, dans un texte intitulé « Les recherches en didactique, les IUFM et le comparatisme en France ». L'auteure y reconnaît que "la création des IUFM (en France) a permis un renforcement des didactiques des disciplines en tant que disciplines de recherche grâce au levier constitué par le recrutement d'enseignants -chercheurs-didacticiens dans chacune des matières scolaires impliquées dans la formation des enseignants, ainsi que l'extension, par voie de conséquence, des communautés scientifiques didactiques » (Amade-Escot, 2013, p. 67). Après une analyse des différents types de travaux qui se sont développés dans ce cadre (optimisation de dispositifs didactiques, analyses de pratiques de classes, etc.), le bilan est plus mitigé quand au développement des recherches comparatistes en didactique : "C'est finalement moins l'existence dans les IUFM de recherches en didactique des disciplines que le choix de certains didacticiens d'endosser une posture scientifique renouvelée, qui peut rendre compte de l'émergence de problématiques comparatistes » (p. 78). Si Chantal AmadeEscot explique cela par le fait que dans l'histoire des sciences, les ruptures épistémologiques sont portées par des chercheurs qui prennent le risque d'introduire et de mettre en débat dans leur communauté, certaines discontinuités ou nécessités nouvelles dans les pratiques de recherche, il n'en reste pas moins que si ces chercheurs ne trouvent pas, à moyen ou long terme, des niches institutionnelles - et donc des ressources- pour déployer leurs programmes de recherche, il y a peu de chance que la rupture épistémologique ait des effets durables sur la manière de structurer le champ de recherche plus globalement.

Ce point de vue, certes, extérieur au dossier, peutêtre mis en regard de l'affirmation d'Yves Reuter qui s'interroge sur « la création de postes de didactique comparée, stratégie potentiellement dangereuse [...] en ce qu'elle peut entrainer le dommage irrémédiable de la perte du travail en commun de spécialistes des différentes disciplines, perspective pourtant stimulante justement ouverte par le projet comparatiste * (Reuter, dans ce numéro). Ce point de vue suscite une réaction de la part de Francia Leutenegger, ellemême titulaire d'une chaire de didactique comparée à l'université de Genève, tout comme Christian Orange à l'Université Libre de Bruxelles. Francia Leutenegger insiste notamment sur la dimension collaborative des recherches de didactique comparée, associant des didacticiens ayant des compétences à propos des disciplines scolaires en jeu. Elle montre également la visée reconstructive de ces recherches, à travers la construction et l'usage d'outils d'analyse à caractère générique, permettant d'établir des rapports entre des faits didactiques distincts, parce qu'enracinés dans des logiques institutionnelles hétérogènes et/ou des constructions disciplinaires différentes (Leutenegger, dans ce numéro).

\section{LES RECRUTEMENTS : TENSION / ARTICULATION ENTRE PROFIL D'ENSEIGNEMENT / FORMATION ET PROFIL DE RECHERCHE}

Les associations professionnelles de chercheurs en didactique(s) sont un atout indéniable dans 
Florence Ligozat et al.

l'évolution du champ, mais on ne peut pas ne pas reconnaître que la construction du champ des recherches en didactique(s) reste fortement dépendante des dynamiques inhérentes aux structures de formation des enseignants. Comment ces structures appréhendent-elles le rapport entre didactique et didactique(s) dans les plans de formation des futurs enseignants ? Quelles sont les valeurs prégnantes dans ces institutions à propos du « métier» de didacticien ?

Dans de nombreux cas, les besoins liés à la demande de formation disciplinaire des enseignants $\mathrm{du}$ primaire et du secondaire représentent des contraintes prioritaires dans le choix des profils de didacticiens à recruter. Ces choix tendent à valoriser les compétences disciplinaires de ces didacticiens plus que leur contribution à la recherche en didactique(s). Il en résulte une forme de sélection qui tend à éliminer les chercheurs - didacticiens qui ne sont pas au bénéfice d'une "formation poussée » (selon la formule de Chevallard, dans ce numéro) dans une discipline apparentée aux disciplines scolaires, ou qui ont malencontreusement une formation initiale dans un domaine peu haut placé dans la hiérarchie des disciplines scolaires ${ }^{9}$. Dans ce contexte, autant dire que le didacticien " gyrovague » qu'Yves Chevallard appelle de ses vœux, promet une situation inconfortable à ceux qui s'y risquent, en regard de la possibilité de trouver un poste dans le fonctionnement actuel des institutions d'accueil des didacticiens. S'affirmer comme un didacticien gyrovague s'annonce difficile, non pour des seules questions épistémologiques de cadres disciplinaires, mais parce que l'univers de la formation professionnelle des enseignants n'a pas encore pris la mesure de ce que le champ des recherches en didactique(s) dans sa globalité, et d'une certaine façon « la didactique », pouvait offrir comme problématisation et contenus de formation, au-delà de la déclinaison des contenus de formation offerts par les didactiques disciplinaires.

Ainsi, la construction du champ des recherches en didactique(s), voire d'une didactique tout court, n'est pas séparable des dynamiques institutionnelles qui animent les institutions d'accueil des recherches, qu'il s'agisse d'instituts ou d'écoles spécifiquement dédiés à la formation des enseignants, de départements universitaires en sciences de l'éducation ou autres disciplines académiques, susceptibles d'accueillir des didacticiens. L'organisation et la structuration des départements comme des unités d'enseignement à l'intérieur des cursus de formation, peut se penser de manière strictement disciplinaire ou au contraire de manière plus mixte. Ces modes d'organisation ont des incidences sur les contours de la profession de « didacticien », et il appartient à ces mêmes didacticiens de veiller aux conditions de développement d'un champ de recherches en didactique(s), dans le contexte mouvant des structures de formation des enseignants.

\section{Linternationalisation de la recherche en éducation}

Au-delà de ces dynamiques liées aux institutions de formation aux métiers de l'enseignement et qui conditionnent l'émergence de certains objets de recherche plutôt que d'autres, Nicole Biagioli souligne l'importance de l'évolution des contraintes qui pèsent sur le monde académique, et en particulier celles liées à l'injonction d'autofinancement : "la course aux appels à projets qui ne concernent que les enjeux économiques, industriels et sociétaux les plus apparents est difficilement compatible avec l'effort conceptuel continu que demande la création et l'amélioration de modèles explicatifs » (Biagioli, dans ce numéro). De nombreux appels d'offres concernant la recherche en éducation relèvent davantage d'enquêtes statistiques sur les effets de telle ou telle réforme ( surveys ) ou de l'élaboration de ressources pour l'enseignement ( capacities) que de la problématisation scientifique des phénomènes liée à l'enseignement et à l'apprentissage. Par l'attractivité financière qu'elles constituent, ces orientations pourraient bien tenir lieu et place de « recherche en didactique(s) » dès lors que des spécificités disciplinaires sont impliquées, alors qu'elles ne reposent souvent que sur la transposition de certains résultats de recherche antérieurement élaborés. C'est peu dire que les didacticiens francophones ont une responsabilité collective majeure dans la mise en place d'une réflexion élargie sur les orientations qu'ils souhaitent donner à la recherche scientifique en didactique(s), dans le contexte d'une internationalisation croissante de la recherche.

Pour certains domaines disciplinaires, des réseaux se sont mis en place dans les années 90, tels que l'European society for Research in Mathematics Éducation (ERME), ou encore l'European Science Éducation Research Association (ESERA) pour ne 
Florence Ligozat et al.

parler que de l'échelle européenne. Au-delà des cadres strictement disciplinaires, le network 27 « Didactics / Teaching \& Learning » de l'European Educational Research Association (EERA) créé en 2006 - la même année que l'ARCD- pose la nécessité d'envisager les rapports entre différentes traditions de recherche sur l'enseignement, l'apprentissage et ses contenus, et notamment les modèles que ces traditions produisent, modèles souvent marqués des contextes socio-politiques distincts (Hudson \& Schneuwly, Éd., 2007 ; Hudson \& Meyer, Éd., 2011). Dans ces divers lieux, l'existence d'une «tradition » de recherche francophone en didactique(s) peut et doit trouver sa place dans la construction d'un espace d'intercompréhension des pratiques d'enseignement et apprentissage au-delà des frontières linguistiques et culturelles des communautés de recherche. Cela ne peut se faire sans une réflexion historique et épistémologique sur les concepts, modèles et méthodes qui sont travaillés régulièrement dans la communauté des didacticiens francophone, dont l'espace de problématisation qui s'ouvre actuellement devrait être porteur.

\section{POUR CONCLURE : LE RÔLE D'UNE POSTURE COMPARATISTE EN DIDACTIQUE}

Nous souhaitons conclure, dans un premier temps, sur l'élaboration de l'espace de problématisation envisagé dans ce texte. Nous avons, à plusieurs endroits, évoqué une possibilité de "crise " pour la (les) didactique(s). Dans le cadre d'une mise en tension des philosophies de John Dewey et de Gaston Bachelard, Michel Fabre (2011) souligne que les « crises » ne sont pas des événements « malheureux », mais des occasions de grandir et de se transformer. À propos de la « crise de l'enseignement », il argumente que l'éducation ne peut plus imposer aux jeunes générations un chemin tout tracé. En s'appuyant sur les métaphores de la carte et de la boussole, il identifie les outils intellectuels, culturels et moraux, que l'enseignement aurait à développer chez les élèves et il évoque l'importance de la problématisation et de l'enquête pour le développement de la pensée scientifique, démarches reconnues comme nécessaire dès la fin du XIX ${ }^{e} \operatorname{siècle~}^{10}$. Pour Michel Fabre, les conditions d'une activité de problématisation font appel à un processus interactif qui peut être modélisé selon quatre axes (invention des conditions - construction et sélection de données - position
- résolution du problème) qui procède de la mise en tension entre un registre empirique de faits et un registre théorique (Fabre \& Orange, 1997). Par extension, qu'est-ce qui pourrait alors être considéré comme un «fait » ou comme un « résultat » didactique?

En sciences, un «fait» se construit dans une relation questionnement-méthode, dans une interaction théorie-pratique et dans une interaction sociale. $\mathrm{La}$ (les) didactique(s), comme science humaine et sociale qui s'interroge sur des phénomènes d'enseignement qui ont d'importants composants stochastiques, doit faire intervenir la confrontation sociale pour valider et coordonner ses « résultats».

Les « résultats » en didactique devraient tendre à l'objectivation et à la validation, certes, mais on constate que peu de travaux sont réellement repris et « discutés » par la communauté de chercheurs. Autrement dit, le champ souffre d'une faible capitalisation de ses travaux ${ }^{11}$. Une posture comparatiste relative aux « résultats " pourrait contribuer à diverses possibilités :

- interroger les résultats par une autre méthodologie ;

- interroger les résultats avec un autre cadre théorique ;

- tester si la même méthodologie conduit aux mêmes résultats.

- Ce qui correspondrait à trois types de validation renvoyant à des domaines différents :

- en termes de reproductibilité ;

- en termes d'interprétation de la construction du résultat ;

- en termes d'objectivité.

Il apparaît important de développer le débat dans la communauté des didacticiens et de favoriser les confrontations, non pour obtenir un consensus à tout prix mais pour susciter une analyse critique constructive.

Dans un second temps, nous sommes donc conduits à souligner des principes d'élaboration ou de renforcement d'une « communauté de didacticiens », au-delà d'éventuelles concurrences ou de certaines formes de polyvalence. Un enjeu pour cette communauté de didacticiens peut être d'examiner différentes modalités d'organisation (ou de réorganisation) du champ des recherches en didactique(s). Plusieurs scénarios, non exclusifs, s'offrent à nous.

Premier scenario : dans le cadre de " la responsabilité vis-à-vis des contenus » rappelée par Jean-Louis 
Florence Ligozat et al.

Martinand, les didactiques ont un intérêt à collaborer étroitement, dans des relations horizontales de complémentarité et de solidarité qui tiennent compte des contraintes des contenus et des modalités scolaires (disciplines, curriculum, mais aussi les différents types d'acteurs des institutions éducatives). Dans ce scénario, le maintien de la diversité des approches didactiques est une priorité.

Deuxième scénario : dans le cadre d'une évolution des organisations scolaires et des pratiques d'enseignement et d'apprentissage, le champ scientifique peut s'enrichir d'une structure favorisant une articulation dialectique entre des didactiques spécifiques et une didactique, entendue comme une "meta-discipline » qui se nourrit des apports des didactiques spécifiques : la didactique " tout court » proposée dans ce numéro par Bernard Schneuwly, une « didactique générale " selon Yves Reuter ou une « didactique disciplinaire généralisée » telle qu'elle se met en place en Allemagne, selon Helmut Johannes Vollmer. Ce processus pourrait être soutenu par une fédération des associations de didactique disciplinaire.

Troisième scénario : dans le cadre d'une transition épistémologique rendue nécessaire par des limitations théorico-méthodologiques émergeant au sein du champ des recherches en didactique(s), un travail de reconstruction conceptuelle peut permettre de faire évoluer ce champ vers une "didactique », envisagée comme une science élaborée autour de la définition d'un noyau commun (la transposition didactique pour Bernard Schneuwly, le système didactique et ses niveaux de détermination, pour Yves Chevallard), pour lesquels des descripteurs se construisent de manière ascendante à partir des spécificités des pratiques d'enseignement et apprentissage, dont il s'agit, entre autres, d'examiner les caractéristiques disciplinaires. C'est plus particulièrement dans cette voie que des travaux de didactique comparée, tels que décrits par Francia Leutenegger dans ce numéro, se sont engagés depuis une dizaine d'années.

Ce dernier scénario peut permettre d'écarter radicalement le risque d'une «solidification» des frontières entre les différents courants de recherches en didactique(s). Dans ce contexte, le didacticien devient d'une certaine manière un anthropologue, c'est-à-dire quelqu'un qui enquête sur la culture, en essayant de comprendre les pratiques humaines de transmission des savoirs, et l'agentivité sise au cœur de ces savoirs, cela grâce à l'élucidation des différences, sur un fond de communauté anthropologique
(Bazin, 2008), entre les pratiques de transmission des savoirs. Mais ce didacticien peut et doit être aussi un ingénieur, qui participe à la production de contenus et de modalités d'apprentissage, ou bien de dispositifs didactiques, ce qui va le conduire à coopérer avec des professeurs et à partager avec eux, localement, temporairement et sous certaines conditions, cette position d'ingénieur. Si l'on peut ainsi envisager un didacticien comme un anthropologue et un ingénieur de la culture, de nouvelles perspectives s'ouvrent à une "science didactique ", aux plans épistémologiques, théoriques, méthodologiques, aux plans éthiques et politiques aussi, en particulier dans le rôle que cette science peut jouer, dans la redéfinition de la profession de professeur, et aussi de celle de chercheur (cf. - par exemple, la structure de l'ingénierie coopérative décrite par Sensevy, Forest, Quilio \& Morales, 2013).

Il y aurait beaucoup à dire sur les contraintes et les possibles liés à chaque scénario ; nous formulons le vœu que le débat puisse se poursuivre dans la communauté des didacticiens. Il nous paraît toutefois indispensable d'insister sur le fait que quelque soit le scénario adopté, si on ne développe pas simultanément une réflexion sur la place de la recherche en didactique(s) dans des structures d'accueil des didacticiens, qui sont trop souvent pilotées par les besoins de formation prioritairement et non par les besoins de la recherche conjointement, la collaboration étroite (scénario 1), l'articulation des didactiques à une (méta)didactique générale (scénario 2), tout comme la (re)construction d'une "science du didactique " du point de vue des pratiques sociales de diffusion des savoirs (scénario 3), ont peu de chance d'aboutir. De notre point de vue, l'élaboration d'un champ de recherche scientifique en didactique(s) passe par la reconnaissance des approches comparatistes et d'une didactique comparée, comme pourvoyeuses d'intelligibilité, de problématisation et de contenus de formation, au même titre que les approches dites " disciplinaires ». La force d'une association telle que l'ARCD est sans doute de pouvoir réunir différents courants de travail comparatistes, afin d'accompagner la (re)structuration du champ des recherches en didactique(s).

Remerciements : nous remercions Chantal AmadeEscot pour sa relecture attentive dans les dernières étapes de l'écriture de ce texte, permettant d'améliorer sa clarté. 
Florence Ligozat et al.

\section{NOTES}

1. «la psychologie».

2. Nous mettons ce complément du nom entre des parenthèses, car la terminologie " les didactiques des disciplines » n'est pas retenue par tous les points de vue dans ce dossier.

3. On peut ainsi citer l'essai d'EIST en France (Enseignement Intégré de Science et de Technologie) ou donner l'exemple de la mise en place du nouveau Plan d'Etude Romand 2011, en Suisse, pour les trois cycles de l'enseignement obligatoire, soit pour les élèves de 4 à 15 ans. Dans ce PER, le domaine « Environnement » pour l'école primaire, qui regroupait des éléments relevant des sciences de la nature et des éléments relevant des sciences sociales, et donc une certaine problématisation des rapports entre nature et culture, a été scindé. Les enseignants de l'école primaire se trouvent désormais face à deux domaines d'étude distincts : celui des « mathématiques et sciences de la nature » articulé autour de la pratique de la modélisation d'une part, et celui des «sciences humaines et sociales " regroupant histoire, géographie, citoyenneté d'autre part.

4. Un concept qui a déjà suscité de longs débats (BlanchardLaville, Chevallard \& Schubauer-Leoni, Éd., 1996)

5. Le développement d'ingénieries didactiques en didactique des mathématiques s'est heurté à ce problème par exemple (Artigue, 2002), comme beaucoup de tentatives d'implantations " descendantes " dans les classes de ressources construites dans le cadre de programmes épistémologiques (pour une discussion sur la différence entre programme épistémologique et programme didactique, cf. Mercier, 2008)

6 . Hofstetter \& Schneuwly (2007) qualifient de « secondarisation disciplinaire", l'institutionnalisation d'un champ d'investigation à propos d'un domaine de pratiques professionnelles normatives.

7. Ligozat \& Leutenegger (2012) montrent que certains profils de didacticiens en Suisse romande résultent d'une migration de chercheurs issus de la psychologie (impliqués dans les psychopédagogies en lien avec les disciplines scolaires) au contact de la montée en puissance des recherches en didactique(s) en France, et du développement de la théorie de la transposition didactique notamment.

8. À un horizon de cinq ans, la grande majorité des chercheurs qui ont contribué à la fondation des didactiques dans le monde francophone, dans les années 1975-1995 ne seront plus (ou peu) actifs.
9. Avoir une formation initiale en musique ou en économie peut permettre d'accéder au rang de didacticien en lien avec les disciplines scolaires apparentées, mais avec le handicap de se confronter à un faible nombre de postes, en comparaison de la situation des chercheurs en didactique des mathématiques, des sciences ou du français. Quant à obtenir un poste de didacticien, après une formation en sciences de l'éducation « tout court », c'est à dire avec une formation qui n'est pas directement apparentée aux disciplines scolaires, les obstacles sont nombreux, même si la personne dispose d'une solide expérience d'enseignement. 10. La démarche d'enquête co-disciplinaire proposée par Yves Chevallard se fait également l'écho de cette préoccupation.

11. Cet aspect également été souligné par Yves Reuter, dans le cadre d’une journée-débat organisée le 27 février 2014 à Genève, suite à la parution de l'ouvrage « Didactique en construction, constructions en didactiques » (Dorier, Leutenegger \& Schneuwly, Éd., 2013) 
Florence Ligozat et al.

\section{RÉFÉRENCES}

Amade-Escot, C. (2013). Les recherches en didactiques, les IUFM et le comparatisme en France. In J.-L. Dorier, F. Leutenegger, \& B. Schneuwly (Éd.), Didactiques en construction, construction de la didactique (Raisons Éducatives, 17, p. 63-83). Bruxelles : De Boeck Université.

Artigue, M. (2002). Ingénierie didactique : quel rôle dans la recherche didactique d'aujourd'hui ? Les Dossiers des Sciences de l'Éducation, Les didactiques scientifiques et technologiques, 8, 59-72.

Bautier, E. Rochex, J.-Y. (2007). Apprendre : des malentendus qui font la différence. In J. Deauvieau \& J.-P. Terail (Éd.), Les sociologues, l'école et la transmission des savoirs, $p$ 227-241. Paris : La Dispute.

Bautier, E. \& Rayou, P. (2013). La littératie scolaire : exigences et malentendus. Les registres du travail des élèves. Éducation E Didactique, 7(2), p 29-46.

Bazin, J. (2008). Des clous dans la Joconde. Lanthropologie autrement. Toulouse : Anacharsis.

Biagioli, N. (2014, ce numéro). Didactique(s) : un singulier-pluriel. Réaction aux points de vue développés par Bernard Schneuwly, Helmut Johannes Vollmer et Yves Chevallard. Éducation \& Didactique, 8(1)

Blanchard-Laville, C., Chevallard, Y., \& Schubauer-Leoni, M.-L. (Éd.). (1996). Regards croisés sur le didactique : un colloque épistolaire. Grenoble: La Pensée sauvage.

Bronckart, J.-P., \& Friedrich, J. (1999/2010). La signification historique de la crise en psychologie. Présentation de l'édition de française. In J.-P. Bronckart \& J. Friedrich (Éd.), L.S. Vygotski. La signification historique de la crise en psychologie. ( $2^{\mathrm{e}}$ éd., p. 15-62). Paris : La Dispute.

Chevallard, Y. (2014, ce numéro). Des didactiques des disciplines scolaires à la didactique comme science anthropologique. Sur un obstacle épistémologique, psychologique et institutionnel. Éducation E Didactique, 8(1).

Chevallard, Y. (1985/1991). La transposition didactique. Du savoir savant au savoir enseigné. ( $2^{\mathrm{e}}$ éd.). Grenoble : La Pensée Sauvage.

Colomb, J., \& Martinand, J.-L. (Éd.). (2000). Éléments pour une didactique comparée, Langue écrite, graphismes et construction des savoirs. Lyon: INRP. Consulté à l'adresse http://www.lcdpu.fr/livre/?GCOI=27000100715130

Daunay, B., Reuter, Y., \& Thépaut, A. (Éd.). (2013). Les contenus disciplinaires : approches comparatistes. Villeneuve d'Ascq : Presses Universitaires du Septentrion.

Dorier, J.-L., Leutenegger, F., \& Schneuwly, B. (Éd.). (2013). Didactique en construction, construction des didactiques. Bruxelles: De Boeck Université.

Elalouf, M.-L., Robert, A., Belhadjin, A., \& Bishop, M.-F. (Éd.). (2012). Les didactiques en questions(s). État des lieux et perspectives pour la recherche et la formation. Bruxelles : De Boeck.

Fabre, M. (2011). Éduquer pour un monde problématique. La carte et la boussole. Paris : PUF

Fabre, M. \& Orange C. (1997). Construction de problèmes et franchissements d'obstacles. Aster 24, 37-57.

Hofstetter, R., \& Schneuwly, B. (2007). Émergence des sciences de l'éducation en Suisse à la croisée de traditions académiques contrastées : fin du $19^{e}$ - première moitié du $20^{e}$ siècle. Bern : Peter Lang.

Hudson, B., \& Meyer, M. A. (Éd.). (2011). Beyond Fragmentation: Didactics, learning and teaching in Europe. Opladen \& Farmington Hills MI : Barbara Budrich Publishers.

Hudson, B., \& Schneuwly, B. (2007). Didactics - learning and teaching in Europe. Editorial. European Educational Research Journal, 6(2), 106-108.

Leutenegger, F. (2014, ce numéro). Didactique et/ou didactiques. Des épistémologies et des postures, des approches et des méthodes. Réaction aux points de vue développés par Yves Reuter \& Jean-Louis Martinand. Éducation \& Didactique, 8(1).

Ligozat, F., \& Leutenegger, F. (2012). Vergleichende Didaktik: Geschichte, Instrumente und Heraufsforderungen aus einer frankophonen Perspektive [Didactique comparée : histoire, outils et enjeux apportés par une perspective francophone]. Pädagogische Rundschau, 66(Heft 6), 751-771.

Martinand, J.-L. (2014, ce numéro). Didactique des sciences et techniques, didactique du curriculum. Éducation E Didactique, 8(1).

Mercier, A. (2008). Pour une lecture anthropologique du programme didactique. Éducation et didactique, 2(1), 7-40.

Mercier, A., Schubauer-Leoni, M. L., \& Sensevy, G. (Éd.). (2002). Vers une didactique comparée. Revue Française de Pédagogie, 141 (numéro spécial). Lyon : INRP.

Orange, C. (2014, ce numéro). Unité et diversité du didactique. Éducation \& Didactique, 8(1).

Raisky, C., \& Caillot, M. (Éd.). (1996). Au-delà des didactiques, le didactique. Débats autour de concepts fédérateurs. Paris: De Boeck Université.

Rayou, P. (2014, ce numéro). Sociologie et didactique. Vers un espace commun de problématisation. Éducation $E$ Didactique, 8(1)

Reuter, Y. (2014, ce numéro). Didactiques et disciplines. Une relation structurelle. Éducation E Didactique, 8(1).

Rocard, M., Csermely, P., Jorde, D., Lenzen, D., WalbergHenriksson, H., \& Hemmo, V. (2007). L'enseignement scientifique aujourd'hui : une pédagogie renouvelée pour l'avenir de l'Europe (No. EUR 22845). Bruxelles : Commission Européenne

Schneuwly, B (2014, ce numéro). Didactique : construction d'un champ disciplinaire. Éducation \& Didactique, 8(1).

Schubauer-Leoni, M. (1988). Le contrat didactique dans une approche psycho-sociale des situations d'enseignement. Interactions didactiques, 8, 63-77.

Sensevy, G., Forest, D., Quilio, S. \& Morales, G. (2013). Cooperative engineering as a specific design-based research. ZDM, The International Journal on Mathematics Éducation, 45(7), 1031-1043

Venturini, P., Amade-Escot, C., \& Terrisse, A. (Éd.). (2002). Étude des pratiques effectives : l'approche des didactiques. Grenoble: La Pensée Sauvage.

Vollmer, H. J. (2014, ce numéro). Fachdidaktik and the developement of generalised subject didactics in Germany. Éducation \& Didactique, 8(1). 